\title{
Encefalitis límbica secundaria a policondritis recidivante
}

\author{
Limbic encephalitis secondary to relapsing polychondritis
}

\author{
Oscar Leonardo Bravo-Ruiz,, \\ Missael De Jesús Salcedo-Hernández, \\ Mario Alberto Mireles-Ramírez, ${ }^{\ddagger}$ Héctor Alberto González-Usigli ${ }^{\ddagger}$
}

\begin{abstract}
RESUMEN. Introducción: La policondritis recidivante (RP) es una enfermedad reumatológica multisistémica. Se estima que 3\% de los pacientes con RP presentan manifestaciones neurológicas, siendo la encefalitis límbica una de las más importantes. La fisiopatología de la encefalitis límbica en RP aún no es del todo comprendida; no obstante, los pacientes que presentan esa manifestación tienen un mal pronóstico de evolución. El objetivo de la presentación del caso clínico es difundir los datos pivote de una manifestación poco frecuente con el fin de recibir tratamiento oportuno. Caso clínico: Masculino de 43 años de edad, diestro, presentó aumento de volumen de ambos pabellones auriculares con hipertermia y dolor a la palpación, posteriormente, pérdida de memoria reciente y cambios conductuales, luego de un mes continuó con afasia y crisis epilépticas acompañadas de movimientos estereotipados orales. Fue abordado bajo el diagnóstico de encefalitis límbica y buscando la causa primaria se hizo hincapié en la exploración física de pabellones auriculares. Conclusiones: La encefalitis límbica en pacientes con RP es poco frecuente; sin embargo, se cuenta con datos pivote para realizar el diagnóstico oportunamente y tratar de delimitar el daño tanto neurológico como sistémico. Conclusión: La encefalitis límbica debe considerarse dentro las manifestaciones secundarias a PR.
\end{abstract}

Palabras clave: Policondritis recidivante, encefalitis límbica, crisis epilépticas, pabellones auriculares, inmunosupresores.

ABSTRACT. Introduction: Relapsing polychondritis. (RP) is a multisystemic rheumatic disease. It is estimated that $3 \%$ of RP patients present neurological manifestations. Being limbic encephalitis one of the most important. The pathophysiology of limbic encephalitis in RP is not yet fully understood, however, patients with this manifestation have a poor prognosis for evolution. The objective of the presentation of the clinical case

* Universidad de Guadalajara, Centro Universitario de Ciencias de la Salud (CUCS), Programa de PNPC CONACyT para la Especialidad de Neurología, Guadalajara, Jalisco, México.

* Unidad Médica de Alta Especialidad, Hospital de Especialidades del Centro Médico de Occidente (CMNO), Departamento de Neurología, Instituto Mexicano del Seguro Social. Guadalajara, Jalisco, México.

$\S$ Médico Cirujano y Partero, Universidad Lamar, Guadalajara, Jalisco, México.
Correspondencia:

Dr. Héctor Alberto González-Usigli

E-mail: hagu73imss@gmail.com

Conflicto de intereses: Es importante aclarar que se cuenta con el consentimiento informado por parte del paciente y no declara inconveniente alguno para divulgación de su caso con fines académicos.

Recibido: 18 de Julio de 2020.

Aceptado: 28 de Agosto de 2020.

Citar como: Bravo-Ruiz OL, García-Ortega YE, Salcedo-Hernández MDJ, Mireles-Ramírez MA, González-Usigli HA. Encefalitis límbica secundaria a policondritis recidivante. El Residente. 2020; 15 (3): 113-117. https://dx.doi.org/10.35366/95961 
is to disseminate the pivotal data of a rare manifestation in order to receive timely treatment. Clinical Case: A 43-year-old male, right-handed, increased volume of both atrial pavilions with hyperthermia and palpation pain, subsequent, recent memory loss and behavioral changes, after 1 month with aphasia and seizures accompanied by stereotyped oral movements, which was approached under the diagnosis of limbic encephalitis and looking for the primary cause, the physical examination of ear pavilions was emphasized. Discussion: Limbic encephalitis in patients with RP is rare, however, there are pivotal data to make the diagnosis in a timely manner and try to delimit the damage both neurologically and systematically. Conclusions: Limbic encephalitis should be included in manifestations secondary to PR.

Keywords: Relapsing polychondritis, limbic encephalitis, epileptic seizures, auricular pavilions, immunosuppressants.

\section{INTRODUCCIÓN}

La policondritis recidivante $(\mathrm{RP})$ es una enfermedad reumatológica multisistémica que se caracteriza por brotes recurrentes de inflamación que afectan el cartílago auricular, nasal, laríngeo y bronquial. ${ }^{1-3}$ Puede presentar afección sistémica, principalmente cardiovascular (24-54\%), ${ }^{4,5}$ se estima que $3 \%$ de los pacientes con RP tiene afección neurológica, y cuando ocurre la convierte en una patología con clínica amplia. ${ }^{6,7}$ Dentro de las principales manifestaciones neuronales en RP se encuentran: neuropatías craneales del quinto y séptimo nervios craneales, ictus, crisis epilépticas, delirio, demencia y encefalitis límbica.

La encefalitis límbica se ha relacionado con pacientes con RP, quienes clínicamente presentan las siguientes manifestaciones: deterioro cognitivo, crisis epilépticas, movimientos anormales estereotipados e involucramientos neuropsiquiátricos tales como depresión, ansiedad, alucinaciones y cambios conductuales, ${ }^{8-11}$ aunque es una presentación inusual de la enfermedad. Una exploración física de pabellones auriculares nos orienta a obtener datos pivotes, y así reposicionar a la RP de ser una de las últimas causas en el abordaje, a nuestra primera impresión diagnóstica, lo que contribuye a prescribir el tratamiento oportuno.

\section{PRESENTACIÓN DEL CASO CLÍNICO}

Paciente masculino de 43 años que acude al servicio de urgencias debido a que desde hace tres meses presentó alteraciones en la memoria reciente y cambios conductuales. Refi- rieron notarlo "tranquilo", fue tratado como una probable encefalopatía de Wernicke sin mejoría tras el tratamiento con complejo B. Dos meses después del evento presentó afasia durante media hora, lo que se atribuyó al diagnóstico de ataque isquémico transitorio, por lo cual se instauró tratamiento a base de ácido acetilsalicílico. Posteriormente presentó un episodio de crisis epilépticas caracterizadas por dextroversión de la mirada y posición tónica de las extremidades superiores con una duración de aproximadamente 20 minutos, acompañadas de pérdida del control de esfínteres, motivo por el cual se decidió inicio de valproato de magnesio, cuatro meses posteriores presentó postura distónica con cuerpo inclinado a la derecha con una duración de minutos, seguida de mioclonías de extremidades superiores, las cuales se autolimitaban en cuestión de minutos. Después de 30 días del evento continuó con otro episodio de las mismas características desencadenado por el desvelo, agregándose disartria posterior al evento y movimientos estereotipados orales. Acude a valoración hospitalaria donde se inicia tratamiento con ceftriaxona a dosis meníngeas y posteriormente aciclovir durante siete días, sin presentar mejoría clínica.

Cuenta con los siguientes antecedentes: carga genética para hipertensión arterial sistémica y diabetes mellitus, tío paterno con enfermedad de Parkinson, consumo de alcohol desde los 16 años, el cual fue suspendido dos años antes del inicio de los síntomas, con un patrón de consumo cada siete días hasta llegar a la embriaguez, consumo de cocaína hace 18 años (en una sola ocasión). 
A su arribo a nuestra unidad se encontró alerta, al estímulo verbal siguió el origen de la voz, y objetos con la mirada, con un habla poco entendible, con lenguaje escrito conservado, obedece órdenes sencillas, pero se distrae con facilidad y tiende a la agresividad. Al realizar la evaluación neurológica encontramos nervios craneales sin alteraciones, motor con tono y trofismo adecuados, fuerza en extremidades no valorado objetivamente debido a poca cooperación del paciente, pero vencía gravedad. Reflejos de estiramiento muscular global ++/++++ así como respuesta plantar indiferente bilateral.

Inicia abordaje diagnóstico con los siguientes estudios:

\begin{tabular}{|c|c|}
\hline Estudio & Resultado \\
\hline Ecocardiograma & Normal \\
\hline Mielocultivo & Sin crecimiento \\
\hline Pruebas de función tiroidea & TSH: 4.02, T4L: 0.94 \\
\hline Anticuerpos antitiroglobulina & 2.2 \\
\hline $\begin{array}{l}\text { PCR para citomegalovirus, } \\
\text { virus herpes y } \\
\text { M. tuberculosis en LCR }\end{array}$ & Negativo \\
\hline Punción lumbar & $\begin{array}{l}\text { Aspecto agua de roca. } \\
\text { Leucocitos: } 0 \text {, glucosa: } \\
\text { 42.5, proteínas: } 58.5 \text {. }\end{array}$ \\
\hline $\begin{array}{l}\text { Cultivos y tinciones } \\
\text { de punción lumbar }\end{array}$ & Negativas \\
\hline Anticuerpos anti-NMDA & Negativo \\
\hline $\begin{array}{l}\text { Antígeno anti-Borrelia burg- } \\
\text { dorferi lgG }\end{array}$ & $\begin{array}{l}<5 \text {, IgM: } 0.3 \\
\text { (ambos negativos) }\end{array}$ \\
\hline
\end{tabular}

Se realizó resonancia magnética, la cual muestra lesiones hiperintensas en núcleo caudado, sustancia blanca e hipocampos no captantes de gadolinio (Figura 1).

Durante su estancia se inició tratamiento empírico con metilprednisolona e inmunoglobulina a una dosis ponderal de $2 \mathrm{~g} / \mathrm{kg}$ sin respuesta favorable debido a la presencia de fiebre. A la exploración física llama la atención la presencia de eritema en pabellones auriculares y aumento de volumen que se limita al hélix, por lo cual se solicitó valoración por reumatología, se realiza el diagnóstico de RP con afección neurológica, se inicia tratamiento con ciclofosfamida, tras su tercer ciclo se observó mejoría, pero con secuelas de epilepsia, reintegrándose a sus actividades laborales y cotidianas dos años después.

Al seguimiento a dos años en consulta externa el paciente continua con epilepsia y deterioro cognitivo leve, aunque es independiente para la mayoría de las actividades de la vida cotidiana.

\section{DISCUSIÓN}

La afección neurológica en RP aparece en aproximadamente $3 \% .{ }^{6}$ Las neuropatías craneales del quinto y séptimo nervios craneales, ictus, crisis epilépticas, delirio, demencia y encefalitis límbica son manifestaciones neurológicas de RP. ${ }^{8}$ El paciente se presentó con datos clínicos de encefalitis límbica por cambios en el comportamiento, crisis epilépticas, deterioro cognitivo y movimientos estereotipados orolinguales aunados a la sospecha clínica de RP, se descartaron otras causas más frecuentes de encefalitis límbica tales como encefalopatía de Hashimoto, lupus eritematoso sistémico del SNC, linfoma intravascular, condiciones paraneoplásicas, postencefalitis herpéticas y encefalitis anti-NMDA. ${ }^{6}$

Hasta un tercio de los pacientes con RP tienen una enfermedad concomitante que incluye: vasculitis sistémica, enfermedad dermatológica, hematológica u otra enfermedad reumática sistémica. ${ }^{12,13}$ La RP no tiene un biomarcador específico; el diagnóstico se establece mediante la variedad de manifestaciones clínicas que se incluyen en los criterios de McAdam's, los cuales son: condritis auricular bilateral, policondritis no erosiva seronegativa, condritis nasal, inflamación ocular, condritis en las vías respiratorias y daño vestibular, el diagnóstico se realiza con un criterio y hallazgos histopatológicos compatibles o la presencia de dos criterios clínicos y respuesta a dosis altas de esteroides o terapia inmunosupresora (como es el caso del paciente). ${ }^{12,13}$

En cuanto al tratamiento no hay muchos estudios que den a conocer el esquema de 

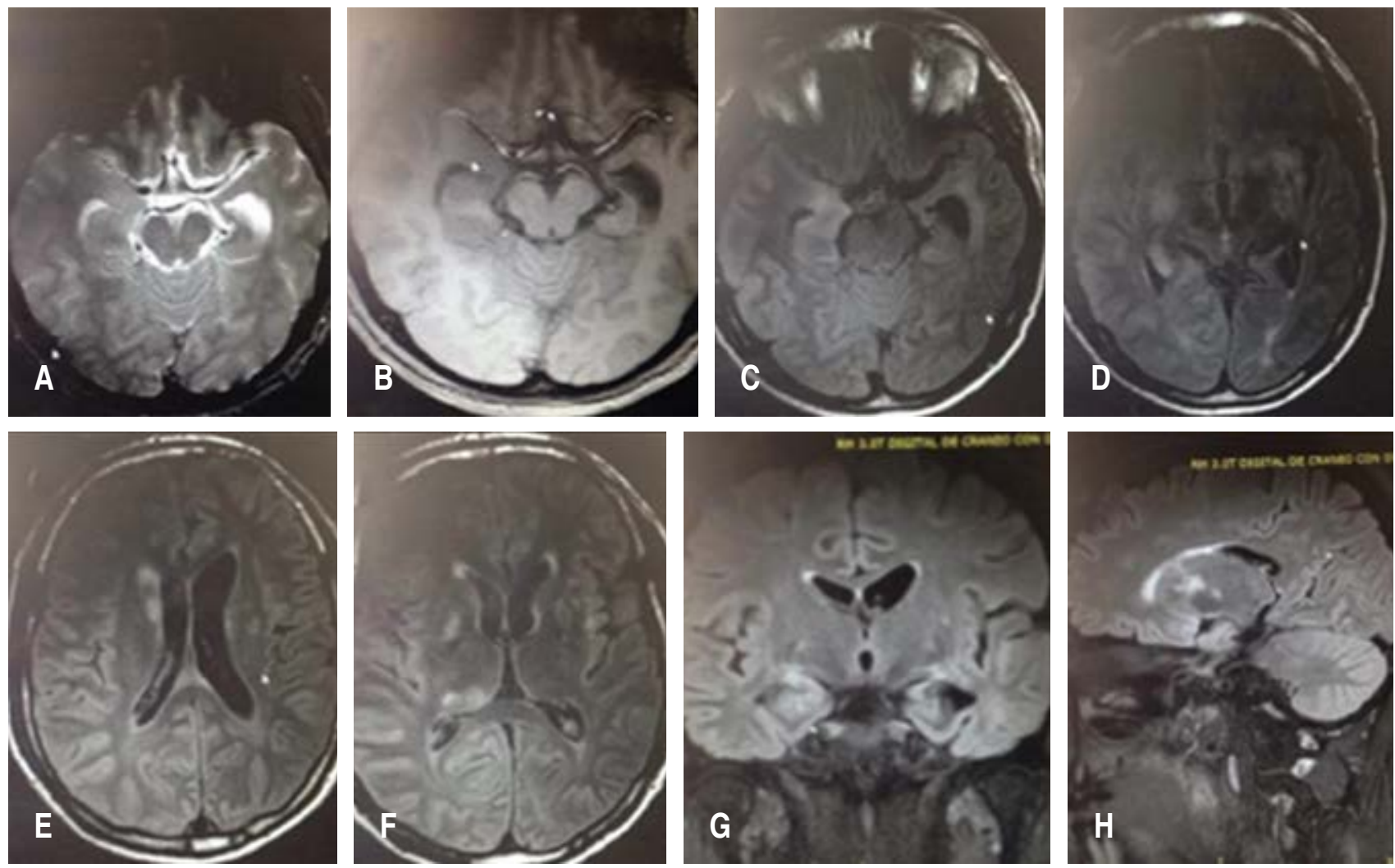

Figura 1: A) Resonancia magnética de encéfalo en la cual se observa hiperintensidad en T2. B-F) FLAIR a nivel de ambos hipocampos y núcleo caudado derecho, redondeadas sin efecto de vector que no presentan captación de gadolinio. G) Corte sagital. H) Coronal en FLAIR donde se observan lesiones hiperintensas en ambos hipocampos, y en corte sagital anteriormente descritas.

tratamiento para encefalitis límbica en RP debido a la baja prevalencia de esta enfermedad. Los esteroides a dosis altas es el tratamiento primordial, en casos refractarios a esteroides se recomienda el uso de otra terapia inmunosupresora o citotóxica como la ciclofosfamida. ${ }^{14,15}$

\section{CONCLUSIÓN}

La encefalitis límbica debe considerarse dentro las manifestaciones secundarias a PR. En este contexto se recomienda incluir la revisión de pabellones auriculares en los pacientes con encefalitis límbica.

\section{BIBLIOGRAFÍA}

1. Puéchal X, Terrier B, Mouthon L, Costedoat-Chalumeau N, Guillevin L, Le Jeunne C. Relapsing polychondritis. Jt Bone Spine. 2014; 81 (2): 118-124. Available from: http:// dx.doi.org/10.1016/j.jbspin.2014.01.001.

2. Mathian A, Miyara M, Cohen-Aubart F, Haroche J, Hie M, Pha M et al. Relapsing polychondritis: a 2016 update on clinical features, diagnostic tools, treatment and biological drug use. Best Pract Res Clin Rheumatol. 2016; 30 (2): 316-333. Available from: http://dx.doi.org/10.1016/j. berh.2016.08.001.

3. Cantarini L, Vitale A, Brizi MG, Caso F, Frediani B, Punzi L et al. Diagnosis and classification of relapsing polychondritis. J Autoimmun. 2014; 48-49: 53-59.
4. Michet CJ Jr, McKenna CH, Luthra HS, O'Fallon WM. Relapsing polychondritis: survival and predictive role of early disease manifestations. Ann Intern Med. 1986; 104: 74-78.

5. McAdam LP, O'Hanlan MA, Bluestone R, Pearson CM. Relapsing polychondritis: prospective study of 23 patients and a review of the literature. Medicine (Baltimore). 1976; 55: 193-213.

6. Hong JC. Relapsing polychondritis with central nervous system involvement: experience of three different cases in a single center. J Korean Med Sci. 2016; 31: 1846-1850. Available from: http://dx.doi.org/10.3346/ jkms.2016.31.11.1846. 
7. Kondo T, Fukuta M, Takemoto A, Takami Y, Sato $\mathrm{M}$, Takahashi $\mathrm{N}$ et al. Limbic encephalitis associated with relapsing polychondritis responded to infliximab and maintained its condition without recurrence after discontinuation: a case report and review of the literature. Ngoya J Med Sci. 2014; 76: 361-368.

8. Hwang YP, Kuo R, Chen TL, Chen PH, Cheng SJ. Relapsing polychondritis presenting with meningoencephalitis and dementia: correlation with neuroimaging and clinical features. Acta Neurol Taiwan. 2015; 24 (1): 30-33.

9. Mathew SD, Battafarano DF, Morris MJ. Relapsing polychondritis in the Department of defense population and review of the literature. Semin Arthritis Rheum. 2012; 42: 70-83.

10. Zeuner M, Straub RH, RauhG, Albert ED, Scholmerich J, Lang B. Relapsing polychondritis: clinical and immunogenetic analysis of 62 patients. J Rheumatol. 1997; 24: 96-101.

11. Sundaram MB, Rajput AH. Nervous system complications of relapsing polychondritis. Neurology. 1983; 33: 513-515.

12. Letko E, Zafirakis P, Baltatzis S, Voudouri A, LivirRallatos C, Foster CS. Relapsing polychondritis: a clinical review. Semin Arthritis Rheum. 2002; 31: 384-395.

13. Frances C, el Rassi R, Laporte JL, Rybojad M, Papo T, Piette JC. Dermatologic manifestations of relapsing polychondritis. A study of 200 cases at a single center. Medicine (Baltimore). 2001; 80: 173-179.

14. Cutolo M, Seriolo B, Pizzorni C, Secchi ME, Soldano $\mathrm{S}$, Paolino $\mathrm{S}$ et al. Use of glucocorticoids and risk of infections. Autoimmun Rev. 2008; 8: 153-155.

15. Stewart KA, Mazenic DJ. Pulse intravenous cyclophosphamide for kidney disease in relapsing polychondritis. J Rheumatol. 1992; 19: 498-500. 